\title{
Die Tagesphänologie epigäischer Spinnen (Arachnida: Araneae) im NSG Hasental-Kregenberg bei Marsberg (NRW)
}

\section{Martin KREUELS}

\begin{abstract}
The daily activity pattern of epigeic spiders (Arachnida: Araneae) in the nature reserve Hasental-Kregenberg near Marsberg (Northrhine-Westfalia, Germany). In a survey of 1,5 years, the daily activity pattern of epigeic spiders was recorded in a xerothermic habitat in the Diemeltal near Marsberg. The correlation between vegetationstructure, daily activity pattern and the hunting type of the spiders is discussed.
\end{abstract}

Key words: epigeic spiders, daily activity pattern, xerothermic habitat, time-sorting pitfall trap, Germany

\section{EINLEITUNG UND ZIELSTELLUNG}

Im Rahmen einer Untersuchung zur tageszeitlichen Aktivität epigäischer Spinnen (KREUELS 1998) wurde eine neukonstruierte tageszeitlich separierendfangende Zeitfalle (KLIEWE 1998) auf einem Kalkmagerrasen (Gentiano-Koelerietum) eingesetzt. Ziel der Untersuchung war die Klärung der Frage, wann die epigäischen Spinnen auf diesem Xerothermstandort ihre Hauptaktivitätsphase(n) haben.

\section{UNTERSUCHUNGSGEBIET}

Die Untersuchung wurde im NSG Hasental-Kregenberg im östlichen Hochsauerland bei Marsberg (Nordrhein-Westfalen) im Diemeltal durchgeführt.

Der Kregenberg gehört der naturräumlichen Untereinheit Marsberger Hochfläche an (SCHULTE 1994). Wie aus dem Namen „NSG HasentalKregenberg" ersichtlich wird, handelt es sich bei dem Naturschutzgebiet um 
zwei Flächen: das Hasental liegt nordwestlich der UF (Untersuchungsfläche) Kregenberg. Es wurde in die Untersuchung nicht mit einbezogen.

Die UF Kregenberg wird an der oberen Hangkante von einem dichten Gebüschsaum begrenzt, der sich über die gesamte Länge der UF erstreckt. Dahinter liegen große Weide- und Ackerflächen. Ein Kiefernwald bildet die westliche Begrenzung des Kalkmagerrasens.

Der Kregenberg ist sowohl aus Schaumkalken des Mittleren Zechsteins, als auch aus kavernösen dolomitischen Kalken des Unteren Zechsteins aufgebaut. Es herrschen Rendzinaböden und Rendzina-Braunerden vor. Die Bodenart ist als steinig-sandiger, teilweise schwach toniger Lehm zu beschreiben.

Tabelle 1: Geographische Angaben zur Lage des Untersuchungsgebietes Tab. 1: Location of the study site

\begin{tabular}{|l|l|}
\hline geographische Lage & NSG Hasenta I-Kregenberg \\
\hline Breite & $51^{\circ} 26^{\prime}, \mathrm{N}$ \\
\hline Länge & $8^{\circ} 52^{\prime}, \mathrm{O}$ \\
\hline TK-Nr. & 4519 \\
\hline Höhe über NN & $295-340 \mathrm{~m}$ \\
\hline Größe der UF & $4 \mathrm{ha}$ \\
\hline Exposition & $\mathrm{SSW}$ \\
\hline Neigung & $22,6^{\circ}$ \\
\hline
\end{tabular}

\section{Falle}

Die Falle wurde vom 03.12.95-11.07.97 eingesetzt. Die Leerungen wurden während des Sommers alle drei Wochen, während des Winters alle sechs Wochen vorgenommen. Das Prinzip der tageszeitlich separierendfangenden Bodenfalle wurde in zahlreichen Publikationen beschrieben (z.B. WILLIAMS 1958, GRANSTROMM 1973, HOLTHAUS \& RIECHERT 1973, PERTERER 1975, BARNDT 1977, TIETZE \& GROSSE 1977, LÖSER 1980, FLATZ, 1987). Auf die Angabe technischer Details wird verzichtet (vgl. dazu KLIEWE 1998). Das Erscheinungsbild der Falle an der Erdoberfläche ähnelt einer herkömmlichen Bodenfalle. Die Öffnung endet in einem $10 \mathrm{I}$ Kunststoffeimer. Innerhalb des Eimers, der vollständig im Boden vergraben 
wird, befindet sich eine motorbetriebene Drehscheibe, auf der sich sechs Fanggefäße (100 mIPE-Flaschen, Fangflüssigkeit: 4\%ige Formalinlösung) befinden. In einem vierstündigen Rhythmus wird ein Gefäß nach dem anderen unter die Einfallöffnung geführt (Dauer der Bewegung: ca. 2 min). Der für den Antrieb benötigte Strom wird durch ein Sonnensegel erzeugt und in eine Autobatterie geleitet. So ist gewährleistet, dass in der Nacht eine ausreichende Stromspannung für den Betrieb des Drehscheibenmotors vorhanden ist. Der nötige Impuls zum Drehen der Scheibe wird von einer Funkuhr gegeben. Die Fangöffnung $(\varnothing 10 \mathrm{~cm}$, Umfang der Fallenöffnung ca. $31 \mathrm{~cm}$ ) ist mit einem Pulvertrichter (Auslaßöffnung $\varnothing 3 \mathrm{~cm}$ ) versehen. Die gesamte Falle ist etwa $40 \mathrm{~cm}$ hoch. Ein Dach $(\varnothing 20 \mathrm{~cm})$ schützt vor Regen und störenden Pflanzenteilen (vgl. HEYDEMANN 1960, ARNOLD 1979).

Die Nomenklatur richtet sich nach PLATNICK ((1997)1998). Die Einordnung in die Lebensweisen der Netzjäger und freijagenden Arten (Tabelle 2) richtet sich nach HOFMANN (1990).

\section{ERGEBNISSE}

Nachfolgend (Tabelle 2) werden alle tagesphänologischen Angaben der Untersuchung aufgeführt. Die Zuordnung der tageszeitlichen Aktivität wurde auf der Grundlage der vorliegenden Daten vorgenommen und berücksichtigt bereits von anderen Autoren publizierte Daten nicht.

Das Verhältnis von Arten, die entweder tag- oder nachtaktiv sind, und Arten, die keiner Phase eindeutig zuzuordnen sind, beträgt 42:23:19. Um die tageszeitliche Aktivität mit der Jagdstrategie zu vergleichen, wurden alle Jagdtypen aufzwei Beuteerwerbsstrategien (Netz-oder Freijäger) reduziert. Das Verhältnis dieser beiden Strategien zueinander betrug 39:45. Es wird deutlich, dass freijagende Jäger überwiegend tagaktiv sind. Ursache hierfür ist wahrscheinlich die visuelle Wahrnehmung der Beute. Zu den tagaktiven Jägern gehören besonders die Gruppen der Lycosiden (excl. Aulonia albimana) und Salticiden, die nahezu ausschließlich optisch jagen. Eine interpretatorische Reduktion auf die tagaktiven Jäger ist hier notwendig, da netzgebundene Jäger wesentlich weniger in Bodenfallen vertreten sind. 
Tabelle 2: Tagesphänologische Angaben der durch die tageszeitlich separierendfangenden Bodenfalle nachgewiesenen Spinnen ( $\mathrm{T}$ : tagaktiv, $\mathrm{N}$ : nachtaktiv)

Tab. 2: Daily activity pattern of epigeic spiders (Arachnida: Araneae) recorded with a timesorting pitfall trap (T: diurnal, N: nocturnal).

\begin{tabular}{|c|c|c|c|c|c|c|c|c|c|c|}
\hline \multirow[b]{2}{*}{ Familie } & \multirow[b]{2}{*}{ Gattung } & \multirow[b]{2}{*}{ Art $(\mathrm{m} / \mathrm{w})$} & \multicolumn{6}{|c|}{ Uhrzeiten der Fangintervalle } & \multirow[b]{2}{*}{ Typ } & \multirow[b]{2}{*}{ Lebensweise } \\
\hline & & & $0-4$ & 4-8 & 8-12 & $12-16$ & $16-20$ & $20-24$ & & \\
\hline \multirow[t]{2}{*}{ Agelenidae } & Agelena & labyrinthica & $1 / 1$ & & & & & & $\mathbf{N}$ & Netz \\
\hline & Tegenaria & silvestris & & & & & & $1 /-$ & $\mathbf{N}$ & Netz \\
\hline Amaurobiidae & Coelotes & terrestris & & $2 /-$ & & & & $2 /-$ & $\mathbf{N}$ & Netz \\
\hline \multirow[t]{2}{*}{ Araneidae } & Hypsosinga & albovittata & & & & $1 /-$ & & & $\mathbf{T}$ & Netz \\
\hline & & sanguinea & & & & -13 & & & $\mathbf{T}$ & Netz \\
\hline \multirow[t]{2}{*}{ Clubionidae } & Cheiracanthium & virescens & -12 & & & & & $-/ 1$ & $\mathbf{N}$ & Frei \\
\hline & Clubiona & diversa & $1 /-$ & $-/ 1$ & & $-/ 1$ & & & $?$ & Frei \\
\hline Dictynidae & Cicurina & cicur & $1 /-$ & $1 /-$ & & & $2 /-$ & $1 /-$ & $\mathbf{N}$ & Netz \\
\hline Dysderidae & Dysdera & erythrina & $1 /-$ & $1 / 1$ & $1 /-$ & & & $2 / 2$ & $\mathbf{N}$ & Frei \\
\hline \multirow[t]{7}{*}{ Gnaphosidae } & Drassodes & cupreus & $9 /-$ & $1 /-$ & & & & $3 /-$ & $\mathbf{N}$ & Frei \\
\hline & & pubescens & $6 / 1$ & $2 /-$ & $1 /-$ & $1 /-$ & & $1 / 1$ & $?$ & Frei \\
\hline & Drassyllus & praeficus & $-/ 1$ & & $3 /-$ & $6 / 3$ & $7 / 1$ & & $T$ & Frei \\
\hline & & pusillus & $-/ 1$ & & $1 /-$ & $3 /-$ & $3 / 1$ & & $T$ & Frei \\
\hline & Haplodrassus & signifer & $1 / 1$ & $-/ 1$ & $1 / 1$ & & & $1 / 1$ & $\mathbf{N}$ & Frei \\
\hline & & umbratilis & $1 / 2$ & & $4 /-$ & & & $2 / 3$ & $?$ & Frei \\
\hline & Zelotes & petrensis & & & & $6 / 4$ & $5 /-$ & & $\mathbf{T}$ & Frei \\
\hline \multirow[t]{2}{*}{ Hahniidae } & Hahnia & helveola & & $2 /-$ & & $1 /-$ & & & $\mathbf{T}$ & Netz \\
\hline & & nava & $-/ 1$ & $1 /-$ & $4 /-$ & 8/- & 9/- & $2 /-$ & $\mathbf{T}$ & Netz \\
\hline \multirow[t]{2}{*}{ Linyphiidae } & Bathyphantes & gracilis & & & $1 /-$ & & & $2 /-$ & $?$ & Netz \\
\hline & & nigrinus & & & & & $1 /-$ & & $\mathbf{T}$ & Netz \\
\hline
\end{tabular}




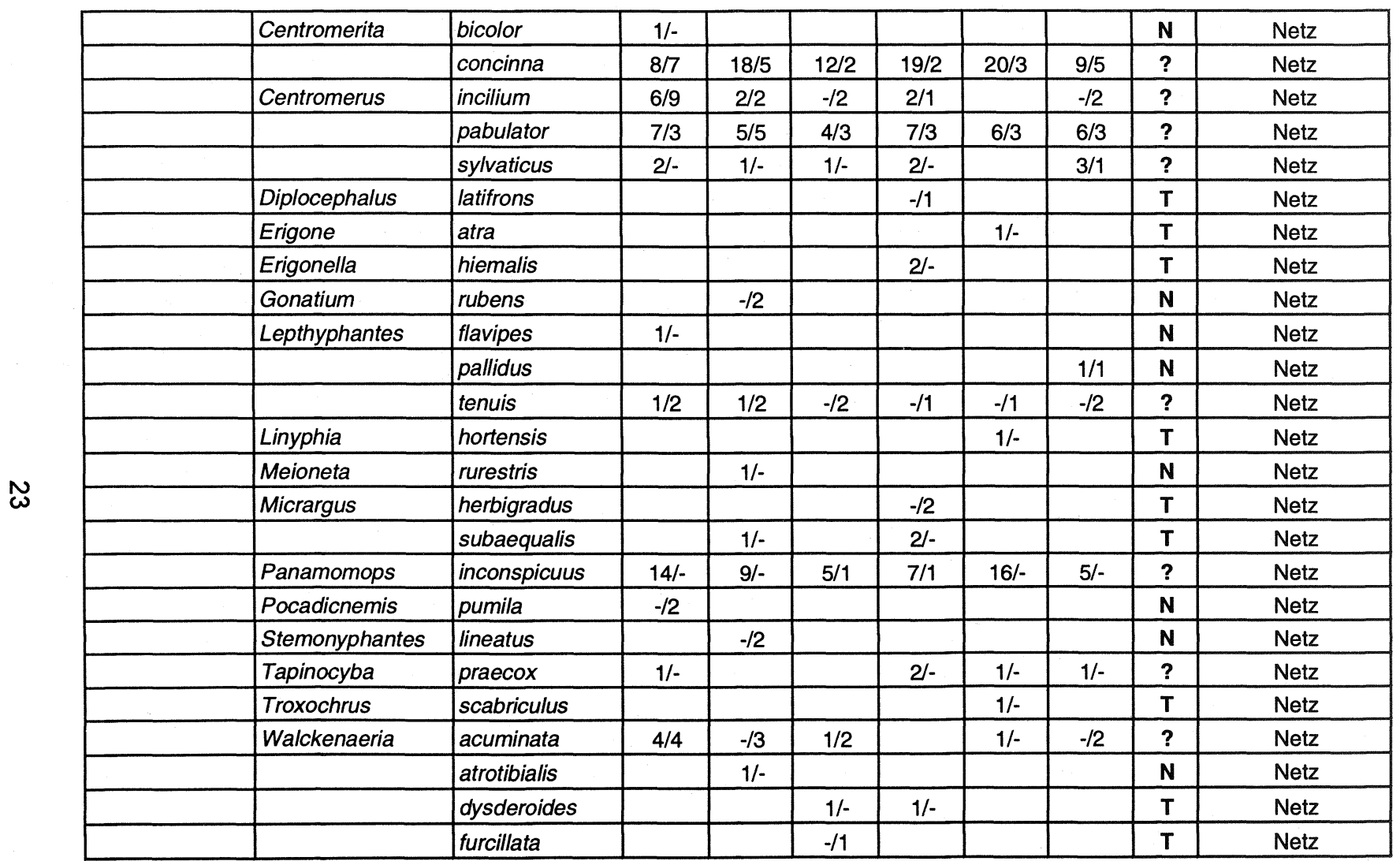


Fortsetzung Tab. 2

\begin{tabular}{|c|c|c|c|c|c|c|c|c|c|c|}
\hline \multirow[b]{2}{*}{ Familie } & \multirow[b]{2}{*}{ Gattung } & \multirow[b]{2}{*}{ Art $(\mathrm{m} / \mathrm{w})$} & \multicolumn{6}{|c|}{ Uhrzeiten der Fangintervalle } & \multirow[b]{2}{*}{ Typ } & \multirow[b]{2}{*}{ Lebensweise } \\
\hline & & & $0-4$ & $4-8$ & $8-12$ & $12-16$ & $16-20$ & $20-24$ & & \\
\hline Liocranidae & Agroeca & brunnea & & $-/ 1$ & & & & & $\mathbf{N}$ & Frei \\
\hline & & cuprea & & 1/- & & & & & $\mathbf{N}$ & Frei \\
\hline & Apostenus & fuscus & $1 /-$ & & $1 /-$ & & $1 / 1$ & $2 /-$ & $?$ & Frei \\
\hline & Micaria & fulgens & & & & $1 / 4$ & $-/ 2$ & & $T$ & Frei \\
\hline & Phrurolithus & festivus & $-/ 1$ & & & & $-/ 1$ & $-/ 1$ & $\mathbf{N}$ & Frei \\
\hline & & minimus & & $1 / 4$ & $2 / 5$ & $1 /-$ & $2 / 2$ & $3 / 3$ & $?$ & Frei \\
\hline & Scotina & celans & & $-/ 1$ & $2 /-$ & $1 / 1$ & & $2 /-$ & $T$ & Frei \\
\hline Lycosidae & Alopecosa & accentuata & & & $6 /-$ & $-/ 1$ & $2 /-$ & & $T$ & Frei \\
\hline & & cuneata & & & $1 /-$ & $6 / 1$ & $2 / 1$ & $2 /-$ & $T$ & Frei \\
\hline & & pulverulenta & & & & $2 /-$ & $1 /-$ & & $T$ & Frei \\
\hline & & trabalis & $3 / 2$ & $1 / 8$ & $14 / 4$ & $31 / 5$ & $46 / 5$ & $9 / 4$ & $T$ & Frei \\
\hline & Arctosa & Iutetiana & $1 /-$ & & & & & $1 /-$ & $\mathbf{N}$ & Frei \\
\hline & Aulonia & albimana & $1 /-$ & $7 / 6$ & $28 / 18$ & $12 / 3$ & $10 / 3$ & $5 / 2$ & $T$ & Netz \\
\hline & Pardosa & amentata & & & & & $-/ 1$ & & $T$ & Frei \\
\hline & & lugubris & & & $1 / 1$ & & $-/ 1$ & & $T$ & Frei \\
\hline & & nigriceps & & & $3 / 3$ & $9 / 1$ & $6 /-$ & $1 /-$ & $T$ & Frei \\
\hline & & palustris & & & & $1 /-$ & & & $T$ & Frei \\
\hline & & pullata & & & $1 / 1$ & $3 / 1$ & & & $T$ & Frei \\
\hline & Trochosa & terricola & $1 /-$ & & & $1 /-$ & & $-/ 1$ & $?$ & Frei \\
\hline & Xerolycosa & nemoralis & & & & & $-/ 1$ & & $T$ & Frei \\
\hline
\end{tabular}




\begin{tabular}{|c|c|c|c|c|c|c|c|c|c|c|}
\hline Mimetidae & Ero & furcata & & $1 /-$ & & & & & $\mathrm{N}$ & Frei \\
\hline Pisauridae & Pisaura & mirabilis & & & & $1 /-$ & $1 /-$ & & $\mathbf{T}$ & Frei \\
\hline \multirow[t]{5}{*}{ Salticidae } & Euophrys & frontalis & & & & $1 /-$ & $-/ 1$ & & $T$ & Frei \\
\hline & Heliophanus & cupreus & & & $1 /-$ & & & $-/ 1$ & $T$ & Frei \\
\hline & & flavipes & & & & & $1 / 1$ & & $\mathrm{~T}$ & Frei \\
\hline & Pellenes & tripunctatus & & & $1 /-$ & & & & $T$ & Frei \\
\hline & Talavera & aequipes & & & & -12 & $-/ 2$ & $-/ 1$ & $T$ & Frei \\
\hline Tetragnathidae & Metellina & segmentata & & & & & $1 /-$ & & $T$ & Netz \\
\hline \multirow[t]{4}{*}{ Theridiidae } & Episinus & truncatus & & & & $1 /-$ & & $-/ 1$ & $?$ & Netz \\
\hline & Euryopis & flavomaculata & $1 /-$ & $1 /-$ & $-/ 1$ & & & $1 /-$ & $?$ & Frei \\
\hline & Robertus & lividus & $1 /-$ & & & & & & $\mathbf{N}$ & Netz \\
\hline & Steatoda & phalerata & & & & $1 /-$ & & & $T$ & Frei \\
\hline \multirow[t]{6}{*}{ Thomisidae } & Ozyptila & atomaria & & & & & $-/ 1$ & $1 /-$ & $\mathbf{N}$ & Frei \\
\hline & & claveata & $1 / 2$ & $-/ 1$ & $14 / 4$ & $10 /-$ & $6 / 2$ & $5 / 1$ & $\mathrm{~T}$ & Frei \\
\hline & & pullata & $1 /-$ & & & $1 /-$ & & $1 /-$ & $?$ & Frei \\
\hline & Xysticus & bifasciatus & & & $2 /-$ & $2 /-$ & $1 /-$ & & $T$ & Frei \\
\hline & & erraticus & & & $1 /-$ & $1 /-$ & & $1 /-$ & $T$ & Frei \\
\hline & & robustus & & & & $1 /-$ & $2 /-$ & & $T$ & Frei \\
\hline Zoriidae & Zora & silvestris & $1 / 2$ & $-/ 1$ & $-/ 1$ & $-/ 1$ & & $-/ 1$ & $?$ & Frei \\
\hline
\end{tabular}




\section{DISKUSSION}

Die Kenntnis der tagesphänologischen Aktivität trägt dazu bei, eine Art einer ökologischen Gruppe zuzuordnen. So ist eine Art, die auf einer wärmebegünstigten trockenen Fläche lebt, nicht zwangsläufig als xerothermophil einzustufen. Verbringt sie die trockenheißen Tagesstunden im Boden oder unter Steinen und weist sie ihr eigentliches Aktivitätsmaximum in den feuchtkühleren Nachtstunden auf, ist sie eher xerothermotolerant, da sie die größte Hitze und Trockenheit inaktiv überdauert. Dagegen ist eine Art auf demselben Standort als xerothermophil einzustufen, wenn ihre tageszeitliche Aktivität in der Zeit der höchsten Temperaturen und größten Trockenheit liegt. Die Diskussion um die zu nutzenden Begriffe muß demnach um das Wissen der tageszeitlichen Aktivität einer Art ergänzt werden. Als Beispiel sei hier Zelotes petrensis genannt: Diese Art ist auf der UF tagaktiv. Dies steht im Gegensatz zur herkömmlichen Auffassung, dass Zelotes-Arten fast ausschließlich nachtaktiv seien (GRIMM 1985). Auch die Aussage von BRAUN (1969), der die von TRETZEL (1954, S. 236) beschriebenen Ansprüche diskutiert, ist zu relativieren. Er schreibt: „[...] aber es sei [...] darauf aufmerksam gemacht, dass die einheimischen Zelotes Arten nächtliche Jäger sind und bei Tage ruhen [...]".

KEER et al. (1989) setzen die tageszeitliche Aktivität mit der Habitatstruktur in Verbindung. Dabei bevorzugen nachtaktive Spinnen hohe und tagaktive Spinnen niedrigwüchsige Vegetationsbereiche. Diese Hypothese wird im wesentlichen durch die vorliegende Untersuchung gestützt. KEER et al. (1989) begründen dies durch die Jagdstrategien der einzelnen Arten. Tagaktive Tiere jagen relativ häufiger auf Sicht, da diese nur in niedrigwüchsiger Vegetation sinnvoll eingesetzt werden kann. Nachtaktive Arten nutzen für die Jagd eher taktile Reize. Die niedrige Vegetation würde bei der letzten Jagdstrategie keine wesentlichen Vorteile bieten.

Die Beziehung aus Habitatstruktur, tageszeitlicher Phänologie und Jagdstrategie ist somit von entscheidener Bedeutung für die Aktivität des Individuums. Eine Interpretation, die ökologische Angaben zu einer Art machen will, und die nur auf einer der drei Faktoren basiert, kann demnach zu fehlerhaften Schlußfolgerungen führen. Das Vorkommen einer Art in einem Lebensraum und ihr Verhalten in diesem Lebensraum ergeben erst Anhaltspunkte zu ihrer ökologischen Einordnung.

Zum Schluß soll noch darauf hingewiesen werden, dass die vorliegenden Daten nicht für andere Standorte in gleicher Weise gelten müssen. So konnten KEER et al. (1989) beobachten, dass Arten an verschiedenen Standorten unterschiedliche tageszeitliche Aktivitäten aufwiesen. Von 
Ähnlichem berichten ebenfalls HENATSCH \& BLICK (1993) und weisen auf unterschiedlich genutzte Beutespektren hin.

\section{ZUSAMMENFASSUNG}

In einer anderthalbjährigen Untersuchung werden tagesphänologische Daten epigeischer Spinnen auf einem xerothermen Standortaufgenommen. Neben diesen Daten werden Angaben zur Jagdstrategie und tageszeitlichen Zugehörigkeit gegeben. Eine Abhängigkeit von Vegetationsstruktur, tageszeitlicher Aktivität und Jagdstrategie und die genaue Begriffsdefinition von xerothermophil oder -tolerant wird diskutiert.

Dank: Herzlich danke ich wieder einmal Heike VOET-KREUELS für die kritischen Anmerkungen. Ebenso bedanke ich mich bei Ralph PLATEN für seine Unterstützung.

\section{LITERATUR}

ARNOLD, K. (1979): Die Verwendung von Schutzvorrichtungen über BARBER-Fallen. 2. Beitrag zur Sammelmethodik von Insekten. - Ent. Ber. 1: 3-6

BARNDT, D. (1977): Untersuchung der diurnalen und saisonalen Aktivität von Käfern mit einer neu entwickelten Elektro-Bodenfalle. - Verh. Bot. Ver. Brandenburg 112: 103-122

BRAUN, R. (1969): Zur Autökologie und Phänologie der Spinnen (Araneida) des Naturschutzgebietes Mainzer Sand. Gleichzeitig ein Beitrag zur Kenntnis der Thermophilie bei Spinnen. - Mainz. Naturwiss. Arch. 8: 193-289

FLATZ, U. (1987): Zur Tagesrhythmik epigäischer Arthropoden (Arachnida, Aranei) einer mesophilen Wiese des Innsbrucker Mittelgebirges (Rinn, $900 \mathrm{~m}$, Nordtirol, Österreich).

- Ber. nat.-med. Verein Innsbruck 74: 159-168

GRANSTROMM, U. (1973): Pitfall traps for studying the activity of groundliving spiders (Araneidae). - Aquilo Ser. Zool. 14: 93-98

GRIMM, U. (1985): Die Gnaphosidae Mitteleuropas (Arachnida, Araneae). - Abh. Naturwiss. Ver. Hamburg 26: 1-318

HENATSCH, B. \& T.BLICK (1993): Zur tageszeitlichen Laufaktivität der Laufkäfer, Kurzflügelkäfer und Spinnen in einer Hecke und einer angrenzenden Brachfläche (Carabidae, Staphylinidae, Araneae). - Mitt. Dtsch. Ges. allge. angew. Ent. 8: 529-536

HEYDEMANN, B. (1960): Über die Bedeutung der Formalinfallen für die zoologische Landesforschung. - Faun. Mitt. 1: 19-24

HOFMANN, I. (1990): Struktur und Sukzession von Spinnengesellschaften der Halbtrockenrasen. - Dissertation Freie Universität Berlin, 193 S.

HOLTHAUS, W.A. \& S.E.RIECHERT (1973): A new time-sort pitfall trap. - Ann. Entomol. Soc. Am. 66: 1362-1364 
KEER, R.de, M.ALDERWEIRELDT, K.DECLEER, H.SEGERS, K.DESENDER \& J.-P. MAELFAIT (1989): Horizontal distribution of the spider fauna of intensively grazed pastures under the influence of diurnal activity and grass height. - J. App. Ent. 107: 455-473

KLIEWE, V. (1998): Elektronisch gesteuerte Zeitfalle zur Untersuchung der tageszeitlichen Aktivität von Bodenarthropoden (Beiträge zur Faunistik und Ökologie der Arthropoden auf den Kalkmagerrasen des Diemeltals, Teil 9). - Beiträge zur Entomologie 48: 541-543

KREUELS, M. (1998): Zur Frage strukturbezogener und phänologischer Anpassungen epigäischer Spinnen (Araneae) auf Kalkmagerrasen im Raum Marsberg. - Dissertation Institut für Landschaftsökologie, Münster, 108 S. \& XXXI S. Anhang.

LÖSER, S. (1980): Zur tageszeitlichen Aktivitätsverteilung von Arthropoda der Bodenstreu (Coleoptera, Diplopoda, Isopoda, Opiliones, Araneae) eines Buchen-Eichen-Waldes (Fago-Quercetum). - Entomologia Generalis 6: 169-180

PERTERER, J. (1975): Macroarthropoden im Grünland der Landesanstalt für Pflanzenzucht und Samenprüfung, Rinn (Nordtirol): Gruppenspektrum, Stratifizierung, Tagesrhythmik. - Hausarbeit Universität Innsbruck, 79 S.

PLATNICK, N.I. ((1997) 1998): Advances in Spider Taxonomy 1992-1995With Redescriptions 1940-1980. New York Entomological Society, New York.

SCHULTE, A.M. (1994): Ökologische Untersuchungen über Heuschrecken im Raum Marsberg. - Diplomarbeit Institut für Landschaftsökologie, Münster, $139 \mathrm{~S}$.

TIETZE, F. \& W.-R.GROSSE (1977): Der zeitfraktionierte Fang von Arthropoden der Bodenoberfläche. - Ent. Nach. Ber. 21: 97-101

TRETZEL, E. (1954): Reife- und Fortpflanzungszeit bei Spinnen. - Z. Morph. Ökol. Tiere 42: $634-691$

WILLIAMS, G. (1958): Mechanical time-sorting of pitfall captures. - J. Animal Ecology 27: $27-35$

AraDet, Dr. Martin KREUELS, Alexander-Hammer-Weg 9, D-48161 Münster e-mail: info@aradet.de 